\title{
Certain Ostrowski type inequalities for generalized s-convex functions
}

\author{
Muharrem Tomar ${ }^{\mathrm{a}}$, Praveen Agarwal ${ }^{\mathrm{b}, \mathrm{c}}$, Mohamed Jleli ${ }^{\mathrm{d}, *}$, Bessem Samet ${ }^{\mathrm{d}}$ \\ ${ }^{a}$ Department of Mathematics, Faculty of Arts and Sciences, Ordu University, 52200 Ordu, Turkey. \\ ${ }^{b}$ Department of Mathematics, Anand International College of Engineering, Jaipur-303012, India. \\ ${ }^{c}$ Department of Mathematics, Ahi Evran University, 40100 Kırşehir-Turkey. \\ ${ }^{d}$ Department of Mathematics, King Saud University, Riyadh-11451, Saudi Arabia.
}

Communicated by S. H. Wu

\begin{abstract}
In this paper, we first obtain a generalized integral identity for twice local differentiable functions. Then, using functions whose second derivatives in absolute value at certain powers are generalized s-convex in the second sense, we obtain some new Ostrowski type inequalities. (C) 2017 All rights reserved.
\end{abstract}

Keywords: Generalized s-convex functions, generalized Hermite-Hadamard inequality, generalized Hölder inequality. 2010 MSC: 26A51, 26A33, 26D10.

\section{Introduction}

Throughout this paper, let $\mathbb{R}, \mathbb{R}^{+}, \mathbb{Q}, \mathbb{Z}$, and $\mathbb{N}$ be the sets of real and positive real numbers, rational numbers, integers, and positive integers, respectively, and

$$
\mathbb{J}:=\mathbb{R} \backslash \mathbb{Q} \text { and } \quad \mathbb{N}_{0}:=\mathbb{N} \cup\{0\} .
$$

In order to describe the definition of the local fractional derivative and local fractional integral, recently, one has introduced the following sets (see, e.g., [19, 22]; see also [4]): for $0<\alpha \leqslant 1$,

(i) the $\alpha$-type set of integers $\mathbb{Z}^{\alpha}$ is defined by

$$
\mathbb{Z}^{\alpha}:=\left\{0^{\alpha}\right\} \cup\left\{ \pm m^{\alpha}: m \in \mathbb{N}\right\}
$$

(ii) the $\alpha$-type set of rational numbers $\mathbb{Q}^{\alpha}$ is defined by

$$
\mathbb{Q}^{\alpha}:=\left\{\mathbf{q}^{\alpha}: \mathrm{q} \in \mathbb{Q}\right\}=\left\{\left(\frac{\mathrm{m}}{\mathrm{n}}\right)^{\alpha}: \mathrm{m} \in \mathbb{Z}, \mathrm{n} \in \mathbb{N}\right\} ;
$$

\footnotetext{
*Corresponding author

Email addresses: muharremtomar@gmail.com (Muharrem Tomar), goyal.praveen2011@gmail.com (Praveen Agarwal), jleli@ksu.edu.sa (Mohamed Jleli), bsamet@ksu.edu.sa (Bessem Samet)

doi:10.22436/jnsa.010.11.32
} 
(iii) the $\alpha$-type set of irrational numbers $\mathbb{J}^{\alpha}$ is defined by

$$
\mathbb{J}^{\alpha}:=\left\{r^{\alpha}: r \in \mathbb{J}\right\}=\left\{r^{\alpha} \neq\left(\frac{m}{n}\right)^{\alpha}: m \in \mathbb{Z}, n \in \mathbb{N}\right\} ;
$$

(iv) the $\alpha$-type set of real line numbers $\mathbb{R}^{\alpha}$ is defined by $\mathbb{R}^{\alpha}:=\mathbb{Q}^{\alpha} \cup \mathbb{J}^{\alpha}$.

Throughout this paper, whenever the $\alpha$-type set $\mathbb{R}^{\alpha}$ of real line numbers is involved, the $\alpha$ is assumed to be tacitly $0<\alpha \leqslant 1$.

One has also defined two binary operations the addition + and the multiplication - (which is conventionally omitted) on the $\alpha$-type set $\mathbb{R}^{\alpha}$ of real line numbers as follows (see, e.g., [19, 22]; see also [4]): for $a^{\alpha}, b^{\alpha} \in \mathbb{R}^{\alpha}$,

Then one finds that

$$
a^{\alpha}+b^{\alpha}:=(a+b)^{\alpha} \text { and } a^{\alpha} \cdot b^{\alpha}=a^{\alpha} b^{\alpha}:=(a b)^{\alpha} .
$$

- $\left(\mathbb{R}^{\alpha},+\right)$ is a commutative group: for $a^{\alpha}, b^{\alpha}, c^{\alpha} \in \mathbb{R}^{\alpha}$,

$\left(\mathrm{A}_{1}\right) \mathrm{a}^{\alpha}+\mathrm{b}^{\alpha} \in \mathbb{R}^{\alpha}$;

$\left(\mathrm{A}_{2}\right) \mathrm{a}^{\alpha}+\mathrm{b}^{\alpha}=\mathrm{b}^{\alpha}+\mathrm{a}^{\alpha}$;

$\left(\mathrm{A}_{3}\right) \mathrm{a}^{\alpha}+\left(\mathrm{b}^{\alpha}+\mathrm{c}^{\alpha}\right)=\left(\mathrm{a}^{\alpha}+\mathrm{b}^{\alpha}\right)+\mathrm{c}^{\alpha}$;

$\left(\mathrm{A}_{4}\right) 0^{\alpha}$ is the identity for $\left(\mathbb{R}^{\alpha},+\right)$ : for any $a^{\alpha} \in \mathbb{R}^{\alpha}, a^{\alpha}+0^{\alpha}=0^{\alpha}+a^{\alpha}=a^{\alpha}$;

$\left(A_{5}\right)$ for each $a^{\alpha} \in \mathbb{R}^{\alpha},(-a)^{\alpha}$ is the inverse element of $a^{\alpha}$ for $\left(\mathbb{R}^{\alpha},+\right)$ :

$$
a^{\alpha}+(-a)^{\alpha}=(a+(-a))^{\alpha}=0^{\alpha} ;
$$

- $\left(\mathbb{R}^{\alpha} \backslash\left\{0^{\alpha}\right\}, \cdot\right)$ is a commutative group: for $\mathrm{a}^{\alpha}, \mathrm{b}^{\alpha}, \mathrm{c}^{\alpha} \in \mathbb{R}^{\alpha}$,

$\left(\mathrm{M}_{1}\right) \mathrm{a}^{\alpha} \mathrm{b}^{\alpha} \in \mathbb{R}^{\alpha}$;

$\left(\mathrm{M}_{2}\right) \mathrm{a}^{\alpha} \mathrm{b}^{\alpha}=\mathrm{b}^{\alpha} \mathrm{a}^{\alpha}$;

$\left(\mathrm{M}_{3}\right) a^{\alpha}\left(b^{\alpha} c^{\alpha}\right)=\left(a^{\alpha} b^{\alpha}\right) c^{\alpha}$;

$\left(M_{4}\right) 1^{\alpha}$ is the identity for $\left(\mathbb{R}^{\alpha}, \cdot\right)$ : for any $a^{\alpha} \in \mathbb{R}^{\alpha}, a^{\alpha} 1^{\alpha}=1^{\alpha} a^{\alpha}=a^{\alpha}$;

$\left(\mathrm{M}_{5}\right)$ for each $\mathrm{a}^{\alpha} \in \mathbb{R}^{\alpha} \backslash\left\{0^{\alpha}\right\},(1 / \mathrm{a})^{\alpha}$ is the inverse element of $\mathrm{a}^{\alpha}$ for $\left(\mathbb{R}^{\alpha}, \cdot\right)$ :

$$
a^{\alpha}(1 / a)^{\alpha}=(a(1 / a))^{\alpha}=1^{\alpha} ;
$$

- Distributive law holds: $a^{\alpha}\left(b^{\alpha}+c^{\alpha}\right)=a^{\alpha} b^{\alpha}+a^{\alpha} c^{\alpha}$.

Furthermore we observe some additional properties for $\left(\mathbb{R}^{\alpha},+, \cdot\right)$ which are stated in the following proposition (see [4]).

Proposition 1.1. Each of the following statements holds true:

(i) like the usual real number system $(\mathbb{R},+, \cdot),\left(\mathbb{R}^{\alpha},+, \cdot\right)$ is a field;

(ii) the additive identity $0^{\alpha}$ and the multiplicative identity $1^{\alpha}$ are unique;

(iii) the additive inverse element and the multiplicative inverse element are unique;

(iv) for each $\mathrm{a}^{\alpha} \in \mathbb{R}^{\alpha}$, its inverse element $(-\mathrm{a})^{\alpha}$ may be written as $-\mathrm{a}^{\alpha}$; for each $\mathrm{b}^{\alpha} \in \mathbb{R}^{\alpha} \backslash\left\{0^{\alpha}\right\}$, its inverse element $(1 / \mathrm{b})^{\alpha}$ may be written as $1^{\alpha} / \mathrm{b}^{\alpha}$ but not as $1 / \mathrm{b}^{\alpha}$;

(v) if the order $<$ is defined on $\left(\mathbb{R}^{\alpha},+, \cdot\right)$ as follows: $\mathrm{a}^{\alpha}<\mathrm{b}^{\alpha}$ in $\mathbb{R}^{\alpha}$ if and only if $\mathrm{a}<\mathrm{b}$ in $\mathbb{R}$, then $\left(\mathbb{R}^{\alpha},+, \cdot,<\right)$ is an ordered field like $(\mathbb{R},+, \cdot,<)$.

In order to introduce the local fractional calculus on $\mathbb{R}^{\alpha}$, we begin with the concept of the local fractional continuity as in Definition 1.2.

Definition 1.2. A non-differentiable function $f: \mathbb{R} \rightarrow \mathbb{R}^{\alpha}, x \mapsto f(x)$, is called to be local fractional continuous at $x_{0}$ if for any $\varepsilon \in \mathbb{R}^{+}$, there exists $\delta \in \mathbb{R}^{+}$such that

$$
\left|f(x)-f\left(x_{0}\right)\right|<\varepsilon^{\alpha}
$$

holds for $\left|x-x_{0}\right|<\delta$. If a function $f$ is local continuous on the interval $(a, b)$, we denote $f \in C_{\alpha}(a, b)$. 
Among several attempts to have defined local fractional derivative and local fractional integral (see [18, Section 2.1]), we choose to recall the following definitions of local fractional calculus (see, e.g., [5, 18, 19]).

Definition 1.3. The local fractional derivative of $f(x)$ of order $\alpha$ at $x=x_{0}$ is defined by

$$
f^{(\alpha)}\left(x_{0}\right)=x_{0} D_{x}^{\alpha} f(x)=\left.\frac{d^{\alpha} f(x)}{d x^{\alpha}}\right|_{x=x_{0}}=\lim _{x \rightarrow x_{0}} \frac{\Delta^{\alpha}\left(f(x)-f\left(x_{0}\right)\right)}{\left(x-x_{0}\right)^{\alpha}},
$$

where $\Delta^{\alpha}\left(f(x)-f\left(x_{0}\right)\right)=\Gamma(\alpha+1)\left(f(x)-f\left(x_{0}\right)\right)$ and $\Gamma$ is the familiar Gamma function (see, e.g., [16, Section 1.1]).

Let $f^{(\alpha)}(x)=D_{x}^{\alpha} f(x)$. If there exists $f^{(k+1) \alpha}(x)=\overbrace{D_{x}^{\alpha} \ldots D_{x}^{\alpha}}^{k+1 \text { times }} f(x)$ for any $x \in I \subseteq \mathbb{R}$, then we denote $f \in D_{(k+1) \alpha}(I)\left(k \in \mathbb{N}_{0}\right)$.

Definition 1.4. Let $f \in C_{\alpha}[a, b]$. Also let $P=\left\{t_{0}, \ldots, t_{N}\right\},(N \in \mathbb{N})$ be a partition of the interval $[a, b]$ which satisfies $a=t_{0}<t_{1}<\cdots<t_{N-1}<t_{N}=b$. Further, for this partition $P$, let $\Delta t:=\max _{0 \leqslant j \leqslant N-1} \Delta t_{j}$ where $\Delta t_{j}:=t_{j+1}-t_{j}\{j=0, \ldots, N-1\}$. Then the local fractional integral of $f$ on the interval $[a, b]$ of order $\alpha$ (denoted by a $\left.\mathrm{I}_{\mathrm{b}}^{(\alpha)} f\right)$ is defined by

$$
{ }_{a} I_{b}^{(\alpha)} f(t)=\frac{1}{\Gamma(\alpha+1)} \int_{a}^{b} f(t)(d t)^{\alpha}:=\frac{1}{\Gamma(\alpha+1)} \lim _{\Delta t \rightarrow 0} \sum_{j=0}^{N-1} f\left(t_{j}\right)\left(\Delta t_{j}\right)^{\alpha},
$$

provided the limit exists (in fact, this limit exists if $f \in C_{\alpha}[a, b]$ ).

Here, it follows that ${ }_{a} \mathrm{I}_{b}^{(\alpha)} f=0$ if $a=b$ and ${ }_{a} I_{b}^{(\alpha)} f=-{ }_{b} I_{a}^{(\alpha)} f$ if $a<b$.

If $\mathrm{I}_{x}^{(\alpha)} \mathrm{g}$ exists for any $x \in[a, b]$ and a function $g:[a, b] \rightarrow \mathbb{R}^{\alpha}$, then we denote $g \in \mathrm{I}_{\chi}^{(\alpha)}[a, b]$.

We give some of the features related to the local fractional calculus that will be required for our main results (see [19]).

Lemma 1.5. The following identities hold true:

(a) (1 $\alpha$-local fractional derivative of $\left.x^{k \alpha}\right)$.

$$
\frac{\mathrm{d}^{\alpha} x^{k \alpha}}{\mathrm{d} x^{\alpha}}=\frac{\Gamma(1+k \alpha)}{\Gamma(1+(k-1) \alpha)} x^{(k-1) \alpha} .
$$

(b) (Local fractional integration is anti-differentiation). Suppose that $\mathrm{f}(\mathrm{x})=\mathrm{g}^{(\alpha)}(\mathrm{x}) \in \mathrm{C}_{\alpha}[\mathrm{a}, \mathrm{b}]$. Then we have

$$
{ }_{a} I_{b}^{\alpha} f(x)=g(b)-g(a) .
$$

(c) (Local fractional integration by parts). Suppose that $f(x), g(x) \in D_{\alpha}[a, b]$ and $f^{(\alpha)}(x), g^{(\alpha)}(x) \in C_{\alpha}[a, b]$. Then we have

$$
{ }_{a} I_{b}^{\alpha} f(x) g^{(\alpha)}(x)=\left.f(x) g(x)\right|_{a} ^{b}-{ }_{a} I_{b}^{\alpha} f^{(\alpha)}(x) g(x)
$$

(d) (Local fractional definite integrals of $x^{\mathrm{k} \alpha}$ ).

$$
\frac{1}{\Gamma(1+\alpha)} \int_{a}^{b} x^{k \alpha}(d x)^{\alpha}=\frac{\Gamma(1+k \alpha)}{\Gamma(1+(k+1) \alpha)}\left(b^{(k+1) \alpha}-a^{(k+1) \alpha}\right) \quad(k \in \mathbb{R}) .
$$

For further details on local fractional calculus, one may refer to [17-21].

Let $I$ be an interval in $\mathbb{R}$. A function $f: I \rightarrow \mathbb{R}^{\alpha}$ is said to be convex on $I$ if

$$
f(t x+(1-t) y) \leqslant t f(x)+(1-t) f(y)
$$

holds for every $x, y \in I$ and $t \in[0,1]$. 
If a function $\mathrm{f}: \mathrm{I} \subset \mathbb{R} \rightarrow \mathbb{R}$ (I an interval) is convex on $\mathrm{I}$, then, for $\mathrm{a}, \mathrm{b} \in \mathrm{I}$ with $\mathrm{a}<\mathrm{b}$, we have

$$
f\left(\frac{a+b}{2}\right) \leqslant \frac{1}{b-a} \int_{a}^{b} f(x) d x \leqslant \frac{f(a)+f(b)}{2},
$$

which is known as the Hermite-Hadamard inequality.

Mo et al. [10] introduced the following generalized convex function.

Definition 1.6. Let $f: I \subset \mathbb{R} \rightarrow \mathbb{R}^{\alpha}$ (I an interval) be a function. If, for any $x_{1}, x_{2} \in I$ and $\lambda \in[0,1]$, the following inequality

$$
f\left(\lambda x_{1}+(1-\lambda) x_{2}\right) \leqslant \lambda^{\alpha} f\left(x_{1}\right)+(1-\lambda)^{\alpha} f\left(x_{2}\right)
$$

holds, then $f$ is called a generalized convex function on $I$.

Here are two basic examples of generalized convex functions:

(1) $f(x)=x^{\alpha p}(p>1)$;

(2) $g(x)=E_{\alpha}\left(x^{\alpha}\right)(x \in \mathbb{R})$, where $E_{\alpha}\left(x^{\alpha}\right):=\sum_{k=0}^{\infty} \frac{x^{\alpha k}}{\Gamma(1+k \alpha)}$ is the Mittag-Leffer function.

Recently the fractal theory has received a significant attention (see, e.g., [1-3, 5-9, 11-15]). Mo et al. [10] proved the following analogue of the Hermite-Hadamard inequality (1.1) for generalized convex functions: let $f(x) \in I_{x}^{\alpha}[a, b]$ be a generalized convex function on $[a, b]$ with $a<b$, then we have

$$
f\left(\frac{a+b}{2}\right) \leqslant \frac{\Gamma(1+\alpha)}{(b-a)^{\alpha}} \text { a } I_{b}^{\alpha} f(x) \leqslant \frac{f(a)+f(b)}{2^{\alpha}} .
$$

Remark 1.7. The double inequality (1.2) is known in the literature as generalized Hermite-Hadamard integral inequality for generalized convex functions. Some of the classical inequalities for means can be derived from (1.2) with appropriate selections of the mapping $f$. Both inequalities in (1.1) and (1.2) hold in the reverse direction if $f$ is concave and generalized concave, respectively. For some more results which generalize, improve, and extend the inequalities (1.2), one may refer to the recent papers $[3,7,9,12-14]$ and references therein.

An analogue in the fractal set $\mathbb{R}^{\alpha}$ of the classical Hölder's inequality has been established by Yang [19], which is asserted by the following lemma.

Lemma 1.8. Let $\mathrm{f}, \mathrm{g} \in \mathrm{C}_{\alpha}[\mathrm{a}, \mathrm{b}]$ with $\frac{1}{\mathrm{p}}+\frac{1}{\mathrm{q}}=1(\mathrm{p}, \mathrm{q}>1)$. Then we have

$$
\frac{1}{\Gamma(\alpha+1)} \int_{a}^{b}|f(x) g(x)|(d x)^{\alpha} \leqslant\left(\frac{1}{\Gamma(\alpha+1)} \int_{a}^{b}|f(x)|^{p}(d x)^{\alpha}\right)^{\frac{1}{p}}\left(\frac{1}{\Gamma(\alpha+1)} \int_{a}^{b}|g(x)|^{q}(d x)^{\alpha}\right)^{\frac{1}{q}} \text {. }
$$

Theorem 1.9 (Generalized Ostrowski inequality). Let $\mathrm{I} \subseteq \mathbb{R}$ be an interval, $\mathrm{f}: \mathrm{I}^{0} \subseteq \mathbb{R} \rightarrow \mathbb{R}^{\alpha}\left(\mathrm{I}^{0}\right.$ is the interior of I) such that $\mathrm{f} \in \mathrm{D}_{\alpha}\left(\mathrm{I}^{0}\right)$, and $\mathrm{f}^{(\alpha)} \in \mathrm{C}_{\alpha}[\mathrm{a}, \mathrm{b}]$ for $\mathrm{a}, \mathrm{b} \in \mathrm{I}^{0}$ with $\mathrm{a}<\mathrm{b}$. Then for all $\mathrm{x} \in[\mathrm{a}, \mathrm{b}]$, we have the identity

$$
\left|f(x)-\frac{\Gamma(1+\alpha)}{(b-a)^{\alpha}} a^{\alpha}{ }_{b}^{\alpha} f(t)\right| \leqslant 2^{\alpha} \frac{\Gamma(1+\alpha)}{\Gamma(1+2 \alpha)}\left[\frac{1}{4^{\alpha}}+\left(\frac{x-\frac{a+b}{2}}{b-a}\right)^{2 \alpha}\right](b-a)^{\alpha}\left\|f^{(\alpha)}\right\|_{\infty} .
$$

In [9], Mo and Sui established the following Hermite-Hadamard inequality for generalized s-convex functions on real linear fractal set $\mathbb{R}^{\alpha}(0<\alpha<1)$.

Theorem 1.10. Suppose that $\mathrm{f}: \mathbb{R}_{+} \rightarrow \mathbb{R}^{\alpha}$ is a generalized s-convex function in the second sense, where $\mathrm{s} \in(0,1)$. Let $\mathrm{a}, \mathrm{b} \in[0, \infty), \mathrm{a}<\mathrm{b}$. If $\mathrm{f} \in \mathrm{C}_{\alpha}[\mathrm{a}, \mathrm{b}]$, then the following inequalities hold:

$$
\frac{2^{(s-1) \alpha}}{\Gamma(1+\alpha)} f\left(\frac{a+b}{2}\right) \leqslant \frac{a I_{b}^{\alpha} f(t)}{(b-a)^{\alpha}} \leqslant \frac{\Gamma(1+s \alpha)}{\Gamma(1+(s+1) \alpha)}(f(a)+f(b)) .
$$

If $\mathrm{f}$ is a generalized s-concave, then we have the reverse inequality. 
In the next section, we first obtain a generalized integral identity for functions twice local differentiable functions. Then, we use this identity to obtain our results and using functions whose second derivatives in absolute value at certain powers are generalized s-convex, to obtain some new Ostrowski type inequalities for functions whose local fractional derivatives are generalized s-convex in the second sense.

\section{Main results}

Lemma 2.1. Let $\mathrm{I} \subseteq \mathbb{R}$ be an interval, $\mathrm{f}: \mathrm{I}^{\circ} \subseteq \mathbb{R} \rightarrow \mathbb{R}$ ( $\mathrm{I}^{\circ}$ is the interior of $\mathrm{I}$ ) such that $\mathrm{f}^{(\alpha)} \in \mathrm{D}_{\alpha}\left(\mathrm{I}^{\circ}\right.$ ) and $\mathrm{f}^{(2 \alpha)} \in \mathrm{C}_{\alpha}[\mathrm{a}, \mathrm{b}]$ for $\mathrm{a}, \mathrm{b} \in \mathrm{I}^{\circ}$ with $\mathrm{a}<\mathrm{b}$. Then, for all $\mathrm{x} \in[\mathrm{a}, \mathrm{b}]$, we have the identity

$$
\begin{aligned}
& \frac{1}{(b-a)^{\alpha}} a_{b}^{\alpha} f(t)-\frac{f(x)}{\Gamma(1+\alpha)}+\frac{(2 x-a-b)^{\alpha} f^{(\alpha)}(x)}{\Gamma(1+2 \alpha)} \\
& =\frac{(x-a)^{3 \alpha}}{\Gamma(1+\alpha) \Gamma(1+2 \alpha)(b-a)^{\alpha}} \int_{0}^{1} t^{2 \alpha} f^{(2 \alpha)}(t x+(1-t) a)(d t)^{\alpha} \\
& \quad+\frac{(b-x)^{3 \alpha}}{\Gamma(1+\alpha) \Gamma(1+2 \alpha)(b-a)^{\alpha}} \int_{0}^{1} t^{2 \alpha} f^{(2 \alpha)}(t x+(1-t) b)(d t)^{\alpha} .
\end{aligned}
$$

Proof. Using the local fractional integration by parts, we have

$$
\begin{aligned}
& \frac{1}{\Gamma(1+\alpha)} \int_{0}^{1} t^{2 \alpha} f^{(2 \alpha)}(t x+(1-t) a)(d t)^{\alpha} \\
&=\left.\frac{t^{2 \alpha}}{(x-a)^{\alpha}} f^{(\alpha)}(t x+(1-t) a)\right|_{0} ^{1}-\frac{\Gamma(1+2 \alpha)}{\Gamma(1+\alpha)\left(x-a^{\alpha}\right)} \frac{1}{\Gamma(1+\alpha)} \int_{0}^{1} t^{\alpha} f^{(\alpha)}(t x+(1-t) a)(d t)^{\alpha} \\
&= \frac{f^{(\alpha)}(x)}{(x-a)^{\alpha}}-\frac{\Gamma(1+2 \alpha)}{(x-a)^{\alpha} \Gamma(1+\alpha)}\left[\left.\frac{t^{\alpha}}{\left(x-a^{\alpha}\right)} f(t x+(1-t) a)\right|_{0} ^{1}\right. \\
&\left.-\frac{\Gamma(1+\alpha)}{(x-a)^{\alpha}} \frac{1}{\Gamma(1+\alpha)} \int_{0}^{1} f(t x+(1-t) a)(d t)^{\alpha}\right] \\
&= \frac{f^{(\alpha)}(x)}{(x-a)^{\alpha}}-\frac{\Gamma(1+2 \alpha) f(x)}{\Gamma(1+\alpha)(x-a)^{2 \alpha}}+\frac{\Gamma(1+2 \alpha)}{(x-a)^{2 \alpha}} \frac{1}{\Gamma(1+\alpha)} \int_{0}^{1} f(t x+(1-t) a)(d t)^{\alpha} .
\end{aligned}
$$

By using the change of the variable $u=t x+(1-t) a$ for $t \in[0,1]$ and by multiplying the both sides of (2.2) by $\frac{(x-a)^{3 \alpha}}{\Gamma(1+2 \alpha)(b-a)^{\alpha}}$, we obtain

$$
\begin{aligned}
& \frac{(x-a)^{3 \alpha}}{\Gamma(1+\alpha) \Gamma(1+2 \alpha)(b-a)^{\alpha}} \int_{0}^{1} t^{2 \alpha} f^{(2 \alpha)}(t x+(1-t) a)(d t)^{\alpha} \\
& =\frac{(x-a)^{2 \alpha} f^{(\alpha)}(x)}{\Gamma(1+2 \alpha)(b-a)^{\alpha}}-\frac{(x-a)^{\alpha} f(x)}{\Gamma(1+\alpha)(b-a)^{\alpha}}+\frac{1}{(b-a)^{\alpha}} a I_{x}^{\alpha} f(t) .
\end{aligned}
$$

Analogously, we also have the following equality:

$$
\begin{aligned}
& \frac{(b-x)^{3 \alpha}}{\Gamma(1+\alpha) \Gamma(1+2 \alpha)(b-a)^{\alpha}} \int_{0}^{1} t^{2 \alpha} f^{(2 \alpha)}(t x+(1-t) b)(d t)^{\alpha} \\
& =-\frac{(b-x)^{2 \alpha} f^{(\alpha)}(x)}{\Gamma(1+2 \alpha)(b-a)^{\alpha}}-\frac{(b-x)^{\alpha} f(x)}{\Gamma(1+\alpha)(b-a)^{\alpha}}+\frac{1}{(b-a)^{\alpha}} \times I_{b}^{\alpha} f(t) .
\end{aligned}
$$

So, adding (2.3) and (2.4), we get desired inequality (2.1). This completes the proof of the lemma. 
Theorem 2.2. Suppose that the assumptions of Lemma 2.1 are satisfied. If $\left|\mathrm{f}^{(2 \alpha)}\right|$ is generalized s-convex in the second sense where $\mathrm{s} \in(0,1)$, then

$$
\begin{aligned}
& \left|\frac{1}{(b-a)^{\alpha}} a_{b}^{\alpha} f(t)-\frac{f(x)}{\Gamma(1+\alpha)}+\frac{(2 x-a-b)^{\alpha} f^{(\alpha)}(x)}{\Gamma(1+2 \alpha)}\right| \\
& \leqslant \frac{(x-a)^{3 \alpha}}{\Gamma(1+2 \alpha)(b-a)^{\alpha}}\left(M(s, \alpha)\left|f^{(2 \alpha)}(x)\right|+N(s, \alpha)\left|f^{(2 \alpha)}(a)\right|\right) \\
& \quad+\frac{(b-x)^{3 \alpha}}{\Gamma(1+2 \alpha)(b-a)^{\alpha}}\left(M(s, \alpha)\left|f^{(2 \alpha)}(x)\right|+N(s, \alpha)\left|f^{(2 \alpha)}(b)\right|\right),
\end{aligned}
$$

where $M(s, \alpha)=\frac{\Gamma(1+(s+2) \alpha)}{\Gamma(1+(s+3) \alpha)}$ and

$$
\mathrm{N}(s, \alpha)=\frac{\Gamma(1+s \alpha)}{\Gamma(1+(s+1) \alpha)}-2^{\alpha} \frac{\Gamma(1+(s+1) \alpha)}{\Gamma(1+(s+2) \alpha)}+\frac{\Gamma(1+(s+2) \alpha)}{\Gamma(1+(s+3) \alpha)}
$$

Proof. Taking modulus in Lemma 2.1 and generalized s-convexity in the second sense of $\left|f^{(2 \alpha)}\right|$, we have

$$
\begin{aligned}
& \left|\frac{1}{(b-a)^{\alpha}} a^{I_{b}^{\alpha}} f(t)-\frac{f(x)}{\Gamma(1+\alpha)}+\frac{(2 x-a-b)^{\alpha} f^{(\alpha)}(x)}{\Gamma(1+2 \alpha)}\right| \\
& \leqslant \frac{(x-a)^{3 \alpha}}{\Gamma(1+\alpha) \Gamma(1+2 \alpha)(b-a)^{\alpha}}\left\{\int_{0}^{1} t^{2 \alpha}\left|f^{(2 \alpha)}(t x+(1-t) a)\right|(d t)^{\alpha}\right. \\
& \left.\quad+\frac{(b-x)^{3 \alpha}}{\Gamma(1+\alpha) \Gamma\left(1+2 \alpha(b-a)^{\alpha}\right.} \int_{0}^{1} t^{2 \alpha}\left|f^{(2 \alpha)}(t x+(1-t) b)\right|(d t)^{\alpha}\right\} \\
& \leqslant
\end{aligned}
$$

Using Lemma 1.5, we also have

$$
\frac{1}{\Gamma(1+\alpha)} \int_{0}^{1} \mathrm{t}^{2 \alpha} \mathrm{t}^{\mathrm{s} \alpha}(\mathrm{dt})^{\alpha}=\frac{\Gamma(1+(\mathrm{s}+2) \alpha)}{\Gamma(1+(\mathrm{s}+3) \alpha)}
$$

and

$$
\frac{1}{\Gamma(1+\alpha)} \int_{0}^{1} t^{2 \alpha}(1-t)^{s \alpha}(d t)^{\alpha}=\frac{\Gamma(1+s \alpha)}{\Gamma(1+(s+1) \alpha)}-2^{\alpha} \frac{\Gamma(1+(s+1) \alpha)}{\Gamma(1+(s+2) \alpha)}+\frac{\Gamma(1+(s+2) \alpha)}{\Gamma(1+(s+3) \alpha)} .
$$

If we substitute equalities (2.7) and (2.8) in (2.6), we get desired inequality (2.5). So, the proof is complete.

Corollary 2.3. In Theorem 2.2 , if we choose $x=\frac{\mathrm{a}+\mathrm{b}}{2}$ and use the s-convexity of $\left|\mathrm{f}^{(2 \alpha)}\right|$, we obtain

$$
\begin{aligned}
& \left|\frac{1}{(b-a)^{\alpha}} a^{\alpha} I_{b}^{\alpha} f(t)-\frac{f\left(\frac{a+b}{2}\right)}{\Gamma(1+\alpha)}\right| \\
& \leqslant \frac{(b-a)^{2 \alpha}}{8^{\alpha} \Gamma(1+2 \alpha)}\left(M(s, \alpha)\left|f^{(2 \alpha)}\left(\frac{a+b}{2}\right)\right|+N(s, \alpha)\left|f^{(2 \alpha)}(a)\right|\right) \\
& \quad+\frac{(b-a)^{2 \alpha}}{8^{\alpha} \Gamma(1+2 \alpha)}\left(M(s, \alpha)\left|f^{(2 \alpha)}\left(\frac{a+b}{2}\right)\right|+N(s, \alpha)\left|f^{(2 \alpha)}(b)\right|\right) \\
& \leqslant \frac{(b-a)^{2 \alpha}}{8^{\alpha} \Gamma(1+2 \alpha)}\left(\frac{2^{(1-s) \alpha} M(s, \alpha)+2^{s \alpha} N(s, \alpha)}{2^{s \alpha}}\right)\left(\left|f^{(2 \alpha)}(a)\right|+\left|f^{(2 \alpha)}(b)\right|\right) .
\end{aligned}
$$


Corollary 2.4. Taking $\Theta:=\left|f^{(2 \alpha)}(x)\right|_{\infty}$ in Theorem 2.2, we get

$$
\begin{aligned}
& \left|\frac{1}{(b-a)^{\alpha}}{ }_{a} I_{b}^{\alpha} f(t)-\frac{f(x)}{\Gamma(1+\alpha)}+\frac{(2 x-a-b)^{\alpha} f^{(\alpha)}(x)}{\Gamma(1+2 \alpha)}\right| \\
& \leqslant \frac{3^{\alpha} \Theta(M(s, \alpha)+N(s, \alpha))}{\Gamma(1+2 \alpha)}\left[\frac{(b-a)^{2 \alpha}}{12^{\alpha}}+\left(x-\frac{a+b}{2}\right)^{2 \alpha}\right] .
\end{aligned}
$$

Corollary 2.5. If we take $\mathrm{x}=\frac{\mathrm{a}+\mathrm{b}}{2}$ in Corollary 2.4, we get

$$
\left|\frac{1}{(b-a)^{\alpha}}{ }_{a} I_{b}^{\alpha} f(t)-\frac{f\left(\frac{a+b}{2}\right)}{\Gamma(1+2 \alpha)}\right| \leqslant \frac{\Theta(M(s, \alpha)+N(s, \alpha))(b-a)^{2 \alpha}}{4^{\alpha} \Gamma(1+2 \alpha)} .
$$

Theorem 2.6. Suppose that the assumptions of Lemma 2.1 are satisfied. If $\left|\mathrm{f}^{(2 \alpha)}\right|^{\mathrm{q}}$ is generalized s-convex in the second sense where $\mathrm{s} \in(0,1)$, then

$$
\begin{aligned}
& \left|\frac{1}{(b-a)^{\alpha}} a_{a} I_{b}^{\alpha} f(t)-\frac{f(x)}{\Gamma(1+\alpha)}+\frac{(2 x-a-b)^{\alpha} f^{(\alpha)}(x)}{\Gamma(1+2 \alpha)}\right| \\
& \leqslant\left(\frac{\Gamma(1+2 p \alpha)}{\Gamma(1+(2 p+1) \alpha)}\right)^{\frac{1}{p}}\left(\frac{\Gamma(1+s \alpha)}{\Gamma(1+(s+1) \alpha)}\right)^{\frac{1}{q}} \frac{1}{\Gamma(1+2 \alpha)(b-a)^{\alpha}} \\
& \quad \times\left[(x-a)^{3 \alpha}\left(\left|f^{(2 \alpha)}(x)\right|^{q}+\left|f^{(2 \alpha)}(a)\right|^{q}\right)^{\frac{1}{q}}\right. \\
& \left.\quad+(b-x)^{3 \alpha}\left(\left|f^{(2 \alpha)}(x)\right|^{q}+\left|f^{(2 \alpha)}(b)\right|^{q}\right)^{\frac{1}{q}}\right],
\end{aligned}
$$

where $\frac{1}{\mathrm{p}}+\frac{1}{\mathrm{q}}=1$.

Proof. Taking modulus Lemma 2.1 and by generalized Hölder inequality, we have

$$
\begin{aligned}
& \left|\frac{1}{(b-a)^{\alpha}} a_{b}^{\alpha} f(t)-\frac{f(x)}{\Gamma(1+\alpha)}+\frac{(2 x-a-b)^{\alpha} f^{(\alpha)}(x)}{\Gamma(1+2 \alpha)}\right| \\
& \leqslant \frac{(x-a)^{3 \alpha}}{\Gamma(1+\alpha) \Gamma(1+2 \alpha)(b-a)^{\alpha}}\left\{\int_{0}^{1} t^{2 \alpha}\left|f^{(2 \alpha)}(t x+(1-t) a)\right|(d t)^{\alpha}\right. \\
& \left.\quad+\frac{(b-x)^{3 \alpha}}{\Gamma(1+\alpha) \Gamma\left(1+2 \alpha(b-a)^{\alpha}\right.} \int_{0}^{1} t^{2 \alpha}\left|f^{(2 \alpha)}(t x+(1-t) b)\right|(d t)^{\alpha}\right\} \\
& \leqslant \\
& \quad \frac{(x-a)^{3 \alpha}}{\Gamma(1+2 \alpha)(b-a)^{\alpha}}\left(\frac{1}{\Gamma(1+\alpha)} \int_{0}^{1} t^{2 p \alpha}(d t)^{\alpha}\right)^{\frac{1}{p}} \times\left(\frac{1}{\Gamma(1+\alpha)} \int_{0}^{1}\left|f^{(2 \alpha)}(t x+(1-t) a)\right|^{q}(d t)^{\alpha}\right)^{\frac{1}{q}} \\
& \quad+\frac{(b-x)^{3 \alpha}}{\Gamma(1+2 \alpha)(b-a)^{\alpha}}\left(\frac{1}{\Gamma(1+\alpha)} \int_{0}^{1} t^{2 p \alpha}(d t)^{\alpha}\right)^{\frac{1}{p}} \times\left(\frac{1}{\Gamma(1+\alpha)} \int_{0}^{1}\left|f^{(2 \alpha)}(t x+(1-t) b)\right|^{q}(d t)^{\alpha}\right)^{\frac{1}{q}} .
\end{aligned}
$$

Since $\left|f^{(2 \alpha)}\right|^{\mathrm{q}}$ is generalized s-convex in the second sense and from generalized Hermite-Hadamard inequality for s-convex functions in the second sense, we have

$$
\begin{aligned}
\int_{0}^{1}\left|f^{(2 \alpha)}(t x+(1-t) a)\right|^{q}(d t)^{\alpha} & =\frac{1}{(x-a)^{\alpha}} \int_{a}^{x}\left|f^{(2 \alpha)}(u)\right|^{q}(d u)^{\alpha} \\
& \leqslant \frac{\Gamma(1+s \alpha)}{\Gamma(1+(s+1) \alpha)}\left(\left|f^{(2 \alpha)}(x)\right|^{q}+\left|f^{(2 \alpha)}(a)\right|^{q}\right)
\end{aligned}
$$


and similarly

$$
\int_{0}^{1}\left|f^{(2 \alpha)}(t x+(1-t) a)\right|^{q}(d t)^{\alpha} \leqslant \frac{\Gamma(1+s \alpha)}{\Gamma(1+(s+1) \alpha)}\left(\left|f^{(2 \alpha)}(x)\right|^{q}+\left|f^{(2 \alpha)}(b)\right|^{q}\right) .
$$

From Lemma 1.5, we also have

$$
\frac{1}{\Gamma(1+\alpha)} \int_{0}^{1} t^{2 p \alpha}(d t)^{\alpha}=\frac{\Gamma(1+2 p \alpha)}{\Gamma(1+(2 p+1) \alpha)}
$$

Now, if we substitute inequalities (2.11), (2.12), and equality (2.13) in (2.10), we obtain

$$
\begin{aligned}
& \left|\frac{1}{(b-a)^{\alpha}}{ }_{a} I_{b}^{\alpha} f(t)-\frac{f(x)}{\Gamma(1+\alpha)}+\frac{(2 x-a-b)^{\alpha} f^{\alpha}(x)}{\Gamma(1+2 \alpha)}\right| \\
& \leqslant\left(\frac{\Gamma(1+2 p \alpha)}{\Gamma(1+(2 p+1) \alpha)}\right)^{\frac{1}{p}}\left(\frac{\Gamma(1+s \alpha)}{\Gamma(1+(s+1) \alpha)}\right)^{\frac{1}{q}} \frac{1}{\Gamma(1+2 \alpha)(b-a)^{\alpha}} \\
& \quad \times\left[(x-a)^{3 \alpha}\left(\left|f^{(2 \alpha)}(x)\right|^{q}+\left|f^{(2 \alpha)}(a)\right|^{q}\right)^{\frac{1}{q}}+(b-x)^{3 \alpha}\left(\left|f^{(2 \alpha)}(x)\right|^{q}+\left|f^{(2 \alpha)}(b)\right|^{q}\right)^{\frac{1}{q}}\right],
\end{aligned}
$$

which is the desired inequality (2.10).

Corollary 2.7. In Theorem 2.6, if we choose $x=\frac{a+b}{2}$ and use the s-convexity of $\left|f^{(2 \alpha)}\right|^{q}$, we get the following inequality:

$$
\begin{aligned}
& \left|\frac{1}{(b-a)^{\alpha}} a I_{b}^{\alpha} f(t)-\frac{f\left(\frac{a+b}{2}\right)}{\Gamma(1+\alpha)}\right| \\
& \leqslant\left(\frac{\Gamma(1+2 p \alpha)}{\Gamma(1+(2 p+1) \alpha)}\right)^{\frac{1}{p}}\left(\frac{\Gamma(1+s \alpha)}{\Gamma(1+(s+1) \alpha)}\right)^{\frac{1}{q}} \frac{(b-a)^{2 \alpha}}{8^{\alpha} \Gamma(1+2 \alpha)} \\
& \times\left[\left(\left|f^{(2 \alpha)}\left(\frac{a+b}{2}\right)\right|^{q}+\left|f^{(2 \alpha)}(a)\right|^{q}\right)^{\frac{1}{q}}+\left(\left|f^{(2 \alpha)}\left(\frac{a+b}{2}\right)\right|^{q}+\left|f^{(2 \alpha)}(b)\right|^{q}\right)^{\frac{1}{q}}\right] \\
& \leqslant\left(\frac{\Gamma(1+2 p \alpha)}{\Gamma(1+(2 p+1) \alpha)}\right)^{\frac{1}{p}}\left(\frac{\Gamma(1+s \alpha)}{\Gamma(1+(s+1) \alpha)}\right)^{\frac{1}{q}} \frac{(b-a)^{2 \alpha}}{8^{\alpha} \Gamma(1+2 \alpha)} \\
& \times\left[\left(\frac{\left(2^{s \alpha}+1\right)\left|f^{(2 \alpha)}(a)\right|^{q}+\left|f^{(2 \alpha)}(b)\right|^{q}}{2^{s \alpha}}\right)^{\frac{1}{q}}+\left(\frac{\left|f^{(2 \alpha)}(a)\right|^{q}+\left(2^{s \alpha}+1\right)\left|f^{(2 \alpha)}(b)\right|^{q}}{2^{s \alpha}}\right)^{\frac{1}{q}}\right] \\
& \leqslant\left(\frac{\Gamma(1+2 p \alpha)}{\Gamma(1+(2 p+1) \alpha)}\right)^{\frac{1}{p}}\left(\frac{\Gamma(1+s \alpha)}{\Gamma(1+(s+1) \alpha)}\right)^{\frac{1}{q}} \\
& \times \frac{\left(1+\left(1+2^{s \alpha}\right)^{\frac{1}{q}}\right)(b-a)^{2 \alpha}}{2^{\left(3+\frac{s}{q}\right) \alpha} \Gamma(1+2 \alpha)}\left(\left|f^{(2 \alpha)}(a)\right|+\left|f^{(2 \alpha)}(b)\right|\right) .
\end{aligned}
$$

While obtaining the last part of the inequality (2.14) it has been used the fact that, for $\mathfrak{u}_{\mathrm{k}}, v_{\mathrm{k}}, 1 \leqslant \mathrm{k} \leqslant \mathrm{n}$ and $0 \leqslant r \leqslant 1$ we have

$$
\sum_{k=1}^{n}\left(u_{k}+v_{k}\right)^{r} \leqslant \sum_{k=1}^{n}\left(u_{k}\right)^{r}+\sum_{k=1}^{n}\left(v_{k}\right)^{r} .
$$

Corollary 2.8. By taking under assumptions of Theorem 2.6 and taking $\Theta:=\left|f^{(2 \alpha)}(x)\right|_{\infty}$, we have 


$$
\begin{aligned}
& \left|\frac{1}{(b-a)^{\alpha}}{ }_{a} I_{b}^{\alpha} f(t)-\frac{f(x)}{\Gamma(1+\alpha)}+\frac{(2 x-a-b)^{\alpha} f^{\alpha}(x)}{\Gamma(1+2 \alpha)}\right| \\
& \quad \leqslant\left(\frac{\Gamma(1+2 p \alpha)}{\Gamma(1+(2 p+1) \alpha)}\right)^{\frac{1}{p}}\left(\frac{\Gamma(1+s \alpha)}{\Gamma(1+(s+1) \alpha)}\right)^{\frac{1}{q}} \frac{3^{\alpha} \Theta}{\Gamma(1+2 \alpha)}\left[\frac{(b-a)^{2 \alpha}}{12^{\alpha}}+\left(x-\frac{a+b}{2}\right)^{2 \alpha}\right] .
\end{aligned}
$$

Corollary 2.9. If we take $\mathrm{x}=\frac{\mathrm{a}+\mathrm{b}}{2}$ in Corollary 2.8 , we get

$$
\left|\frac{1}{(b-a)^{\alpha}}{ }_{a} I_{b}^{\alpha} f\left(\frac{a+b}{2}\right)-\frac{f(x)}{\Gamma(1+\alpha)}\right| \leqslant\left(\frac{\Gamma(1+2 p \alpha)}{\Gamma(1+(2 p+1) \alpha)}\right)^{\frac{1}{p}}\left(\frac{\Gamma(1+s \alpha)}{\Gamma(1+(s+1) \alpha)}\right)^{\frac{1}{q}} \frac{\Theta(b-a)^{2 \alpha}}{4^{\alpha} \Gamma(1+2 \alpha)} .
$$

Theorem 2.10. Suppose that the assumptions of Lemma 2.1 are satisfied. If $\left|f^{(2 \alpha)}\right|^{q}$ is generalized s-convex in the second sense where $\mathrm{s} \in(0,1)$, then for all $\mathrm{q} \geqslant 1$

$$
\begin{aligned}
& \left|\frac{1}{(b-a)^{\alpha}} a^{\alpha} I_{b}^{\alpha} f(t)-\frac{f(x)}{\Gamma(1+\alpha)}+\frac{(2 x-a-b)^{\alpha} f^{(\alpha)}(x)}{\Gamma(1+2 \alpha)}\right| \\
& \leqslant\left(\frac{\Gamma(1+2 \alpha)}{\Gamma(1+3 \alpha)}\right)^{1-\frac{1}{q}} \frac{1}{\Gamma(1+2 \alpha)(b-a)^{\alpha}} \\
& \quad \times\left[(x-a)^{3 \alpha}\left(M(s, \alpha)\left|f^{(2 \alpha)}(x)\right|^{q}+N(s, \alpha)\left|f^{(2 \alpha)}(a)\right|^{q}\right)^{\frac{1}{q}}\right. \\
& \left.\quad+(b-x)^{3 \alpha}\left(M(s, \alpha)\left|f^{(2 \alpha)}(x)\right|^{q}+N(s, \alpha)\left|f^{(2 \alpha)}(b)\right|^{q}\right)^{\frac{1}{q}}\right] .
\end{aligned}
$$

Proof. From Lemma 2.1 and by generalized power-mean inequality, we have

$$
\begin{aligned}
& \left|\frac{1}{(b-a)^{\alpha}}{ }_{a} I_{b}^{\alpha} f(t)-\frac{f(x)}{\Gamma(1+\alpha)}+\frac{(2 x-a-b)^{\alpha} f^{(\alpha)}(x)}{\Gamma(1+2 \alpha)}\right| \\
& \leqslant \frac{(x-a)^{3 \alpha}}{\Gamma(1+\alpha) \Gamma(1+2 \alpha)(b-a)^{\alpha}}\left\{\int_{0}^{1} t^{2 \alpha}\left|f^{(2 \alpha)}(t x+(1-t) a)\right|(d t)^{\alpha}\right. \\
& \left.\quad+\frac{(b-x)^{3 \alpha}}{\Gamma(1+\alpha) \Gamma(1+2 \alpha)(b-a)^{\alpha}} \int_{0}^{1} t^{2 \alpha}\left|f^{(2 \alpha)}(t x+(1-t) b)\right|(d t)^{\alpha}\right\} \\
& \leqslant \\
& \quad \frac{(x-a)^{3 \alpha}}{\Gamma(1+2 \alpha)(b-a)^{\alpha}}\left(\frac{1}{\Gamma(1+\alpha)} \int_{0}^{1} t^{2 \alpha}(d t)^{\alpha}\right)^{1-\frac{1}{q}} \\
& \quad+\frac{\left(\frac{1}{\Gamma(1+\alpha)} \int_{0}^{1} t^{2 \alpha} \mid f^{(2 \alpha)}(t x+(1-t) a)^{3 \alpha}(d t)^{\alpha}\right)^{\frac{1}{q}}}{\Gamma(1+2 \alpha)(b-a)^{\alpha}}\left(\frac{1}{\Gamma(1+\alpha)} \int_{0}^{1} t^{2 \alpha}(d t)^{\alpha}\right)^{1-\frac{1}{q}} \\
& \quad \times\left(\frac{1}{\Gamma(1+\alpha)} \int_{0}^{1} t^{2 \alpha}\left|f^{(2 \alpha)}(t x+(1-t) b)\right|^{q}(d t)^{\alpha}\right)^{\frac{1}{q}} .
\end{aligned}
$$

Since $\left|f^{(2 \alpha)}\right|^{\mathrm{q}}$ is generalized s-convex in the second sense, we have

$$
\left|f^{(2 \alpha)}(t x+(1-t) a)\right|^{q} \leqslant t^{s \alpha}\left|f^{(2 \alpha)}(x)\right|^{q}+(1-t)^{s \alpha}\left|f^{(2 \alpha)}(a)\right|^{q}
$$

and

$$
\left|f^{(2 \alpha)}(t x+(1-t) b)\right|^{q} \leqslant t^{s \alpha}\left|f^{(2 \alpha)}(x)\right|^{q}+(1-t)^{s \alpha}\left|f^{(2 \alpha)}(b)\right|^{q} .
$$


Thus we can write,

$$
\begin{aligned}
& \mid \frac{1}{(b-a)^{\alpha}} \text { a } I_{b}^{\alpha} f(t)-\frac{f(x)}{\Gamma(1+\alpha)}+\frac{(2 x-a-b)^{\alpha} f^{(\alpha)}(x)}{\Gamma(1+2 \alpha)} \mid \\
& \leqslant \frac{(x-a)^{3 \alpha}}{\Gamma(1+2 \alpha)(b-a)^{\alpha}}\left(\frac{1}{\Gamma(1+\alpha)} \int_{0}^{1} t^{2 \alpha}(d t)^{\alpha}\right)^{1-\frac{1}{q}} \\
& \quad \times\left(\frac{1}{\Gamma(1+\alpha)} \int_{0}^{1} t^{2 \alpha}\left[t^{s \alpha}\left|f^{(2 \alpha)}(x)\right|^{q}+(1-t)^{s \alpha}\left|f^{(2 \alpha)}(a)\right|^{q}\right](d t)^{\alpha}\right)^{\frac{1}{q}} \\
& \quad+\frac{(b-x)^{3 \alpha}}{\Gamma(1+2 \alpha)(b-a)^{\alpha}}\left(\frac{1}{\Gamma(1+\alpha)} \int_{0}^{1} t^{2 \alpha}(d t)^{\alpha}\right)^{1-\frac{1}{q}} \\
& \quad \times\left(\frac{1}{\Gamma(1+\alpha)} \int_{0}^{1} t^{2 \alpha}\left[t^{s \alpha}\left|f^{(2 \alpha)}(x)\right|^{q}+(1-t)^{s \alpha}\left|f^{(2 \alpha)}(b)\right|^{q}\right](d t)^{\alpha}\right)^{\frac{1}{q}} .
\end{aligned}
$$

Using Lemma 1.5, we have

$$
\begin{gathered}
\frac{1}{\Gamma(1+\alpha)} \int_{0}^{1} \mathrm{t}^{2 \alpha}(\mathrm{dt})^{\alpha}=\frac{\Gamma(1+2 \alpha)}{\Gamma(1+3 \alpha)^{\prime}} \\
\frac{1}{\Gamma(1+\alpha)} \int_{0}^{1} \mathrm{t}^{2 \alpha} \mathrm{t}^{\mathrm{s} \alpha}(\mathrm{dt})^{\alpha}=\frac{\Gamma(1+(\mathrm{s}+2) \alpha)}{\Gamma(1+(\mathrm{s}+3) \alpha)},
\end{gathered}
$$

and

$$
\frac{1}{\Gamma(1+\alpha)} \int_{0}^{1} t^{2 \alpha}(1-t)^{s \alpha}(d t)^{\alpha}=\frac{\Gamma(1+(s+2) \alpha)}{\Gamma(1+(s+3) \alpha)}-2^{\alpha} \frac{\Gamma(1+(s+1) \alpha)}{\Gamma(1+(s+2) \alpha)}+\frac{\Gamma(1+s \alpha)}{\Gamma(1+(s+1) \alpha)} .
$$

Substituting (2.17), (2.18), and (2.19) in (2.16), we get desired inequality (2.15). So the proof of this theorem is complete.

Corollary 2.11. In Theorem 2.10, if we choose $x=\frac{\mathrm{a}+\mathrm{b}}{2}$ and use the s-convexity of $\left|\mathrm{f}^{(2 \alpha)}\right|^{\mathrm{q}}$, we get the following inequality:

$$
\begin{aligned}
\left|\frac{1}{(b-a)^{\alpha}}{ }_{a} I_{b}^{\alpha} f(t)-\frac{f\left(\frac{a+b}{2}\right)}{\Gamma(1+\alpha)}\right| \leqslant & \left(\frac{\Gamma(1+2 \alpha)}{\Gamma(1+3 \alpha)}\right)^{1-\frac{1}{q}} \frac{(b-a)^{2 \alpha}}{8^{\alpha} \Gamma(1+2 \alpha)} \\
& \times\left[\left(M(s, \alpha)\left(\frac{\left|f^{(2 \alpha)}(a)\right|+\left|f^{(2 \alpha)}(b)\right|}{2^{s \alpha}}\right)+N(s, \alpha)\left|f^{(2 \alpha)}(a)\right|\right)^{\frac{1}{q}}\right. \\
& \left.+\left(M(s, \alpha)\left(\frac{\left|f^{(2 \alpha)}(a)\right|+\left|f^{(2 \alpha)}(b)\right|}{2^{s \alpha}}\right)+N(s, \alpha)\left|f^{(2 \alpha)}(b)\right|\right)^{\frac{1}{q}}\right] \\
\leqslant & \left(\frac{\Gamma(1+2 \alpha)}{\Gamma(1+3 \alpha)}\right)^{1-\frac{1}{q}} \frac{(b-a)^{2 \alpha}}{2^{\left(3+\frac{s}{q}\right) \alpha} \Gamma(1+2 \alpha)} \\
& \times\left(\left(M(s, \alpha)+2^{s \alpha} N(s, \alpha)\right)^{\frac{1}{q}}+M(s, \alpha)^{\frac{1}{q}}\right)\left(\left|f^{(2 \alpha)}(a)\right|+\left|f^{(2 \alpha)}(b)\right|\right) .
\end{aligned}
$$

Corollary 2.12. By the assumptions of Theorem 2.10 and taking $\Theta:=\left|f^{(2 \alpha)}(x)\right|_{\infty}$, we have 


$$
\begin{aligned}
& \left|\frac{1}{(b-a)^{\alpha}} a^{\alpha} f(t)-\frac{f\left(\frac{a+b}{2}\right)}{\Gamma(1+\alpha)}+\frac{(2 x-a-b)^{\alpha} f^{\alpha}(x)}{\Gamma(1+2 \alpha)}\right| \\
& \quad \leqslant\left(\frac{\Gamma(1+2 \alpha)}{\Gamma(1+3 \alpha)}\right)^{1-\frac{1}{q}} \frac{3^{\alpha} \Theta(M(s, \alpha)+N(s, \alpha))^{\frac{1}{q}}}{\Gamma(1+2 \alpha)}\left[\frac{(b-a)^{2 \alpha}}{12^{\alpha}}+\left(x-\frac{a+b}{2}\right)^{2 \alpha}\right] .
\end{aligned}
$$

Corollary 2.13. If we take $\mathrm{x}=\frac{\mathrm{a}+\mathrm{b}}{2}$ in Corollary 2.12, we get

$$
\left|\frac{1}{(b-a)^{\alpha}} a^{\alpha}{ }_{b}^{\alpha} f(t)-\frac{f\left(\frac{a+b}{2}\right)}{\Gamma(1+\alpha)}\right| \leqslant\left(\frac{\Gamma(1+2 \alpha)}{\Gamma(1+3 \alpha)}\right)^{1-\frac{1}{q}} \frac{\Theta(M(s, \alpha)+N(s, \alpha))^{\frac{1}{q}}(b-a)^{2 \alpha}}{4^{\alpha} \Gamma(1+2 \alpha)} .
$$

\section{Acknowledgment}

The third and the fourth authors extend their appreciation to the Deanship of Scientific Research at King Saud University for funding this work through research group No RGP-237.

\section{References}

[1] P. Agarwal, Some inequalities involving Hadamard-type k-fractional integral operators, Math. Methods Appl. Sci., 40 (2017), 3882-3891. 1

[2] P. Agarwal, M. Jleli, M. Tomar, Certain Hermite-Hadamard type inequalities via generalized k-fractional integrals, J. Inequal. Appl., 2017 (2017), 10 pages.

[3] H. Budak, M. Z. Sarikaya, H. Yildirim, New inequalities for local fractional integrals, RGMIA Research Report Collection, 18 (2015), 13 pages. 1, 1.7

[4] J.-S. Choi, E. Set, M. Tomar, Certain generalized Ostrowski type inequalities for local fractional integrals, Commun. Korean Math. Soc., 32 (2017), 601-617. 1, 1

[5] S. Erden, M. Z. Sarikaya, Generalized Pompeiu type inequalities for local fractional integrals and its applications, Appl. Math. Comput., 274 (2016), 282-291. 1, 1

[6] M. A. Latif, Inequalities of Hermite-Hadamard type for functions whose derivatives in absolute value are convex with applications, Arab J. Math. Sci., 21 (2015), 84-97.

[7] H.-X. Mo, Generalized Hermite-Hadamard inequalities involving local fractional integral, ArXiv, 2014 (2014), 8 pages. 1.7

[8] H.-X. Mo, X. Sui, Generalized s-convex functions on fractal sets, Abstr. Appl. Anal., 2014 (2014), 8 pages.

[9] H.-X. Mo, X. Sui, Hermite-Hadamard-type inequalities for generalized s-convex functions on real linear fractal set $\mathbb{R}^{\alpha}$ $(0<\alpha<1)$, Math. Sci. (Springer), 11 (2017), 241-246. 1, 1.7, 1

[10] H.-X. Mo, X. Sui, D.-Y. Yu, Generalized convex functions on fractal sets and two related inequalities, Abstr. Appl. Anal., 2014 (2014), 7 pages. 1, 1

[11] S. K. Ntouyas, P. Agarwal, J. Tariboon, On Pólya-Szegö and Chebyshev types inequalities involving the Riemann-Liouville fractional integral operators, J. Math. Inequal., 10 (2016), 491-504. 1

[12] M. Z. Sarikaya, H. Budak, Generalized Ostrowski type inequalities for local fractional integrals, Proc. Amer. Math. Soc., 145 (2017), 1527-1538. 1.7

[13] M. Z. Sarikaya, S. Erden, H. Budak, Some generalized Ostrowski type inequalities involving local fractional integrals and applications, RGMIA Research Report Collection, 18 (2015), 12 pages.

[14] M. Z. Sarikaya, T. Tunc, H. Budak, On generalized some integral inequalities for local fractional integrals, Appl. Math. Comput., 276 (2016), 316-323. 1.7

[15] E. Set, M. Tomar, New inequalities of Hermite-Hadamard type for generalized convex functions with applications, Facta Univ. Ser. Math. Inform., 31 (2016), 383-397. 1

[16] H. M. Srivastava, J.-S. Choi, Zeta and q-Zeta functions and associated series and integrals, Elsevier, Inc., Amsterdam, (2012). 1.3

[17] X.-J. Yang, Generalized local fractional Taylor's formula with local fractional derivative, ArXiv, 2011 (2011), 5 pages. 1

[18] X.-J. Yang, Local fractional functional analysis and its applications, Asian Academic publisher Limited, Hong Kong, (2011). 1

[19] X.-J. Yang, Advanced local fractional calculus and its applications, World Science Publisher, New York, (2012). 1, 1, 1, 1

[20] X.-J. Yang, Local fractional Fourier analysis, Adv. Mech. Eng. Appl., 1 (2012), 12-16.

[21] X.-J. Yang, Local fractional integral equations and their applications, Adv. Comput. Sci. Appl., 1 (2012), 234-239. 1

[22] Y.-J. Yang, D. Baleanu, X.-J. Yang, Analysis of fractal wave equations by local fractional Fourier series method, Adv. Math. Phys., 2013 (2013), 6 pages. 1 